TRANSACTIONS OF THE

AMERICAN MATHEMATICAL SOCIETY

Volume 00, Number 0, Pages 000-000

S $0002-9947(\mathrm{XX}) 0000-0$

\title{
SOLVABLE LOCAL AND STOCHASTIC VOLATILITY MODELS: SUPERSYMMETRIC METHODS IN OPTION PRICING
}

\author{
PIERRE HENRY-LABORDÈRE
}

\begin{abstract}
In this paper we provide an extensive classification of one and two dimensional diffusion processes which admit an exact solution to the Kolmogorov (and hence Black-Scholes) equation (in terms of hypergeometric functions). By identifying the one-dimensional solvable processes with the class of integrable superpotentials introduced recently in supersymmetric quantum mechanics, we obtain new analytical solutions. In particular, by applying supersymmetric transformations on a known solvable diffusion process (such as the Natanzon process for which the solution is given by a hypergeometric function), we obtain a hierarchy of new solutions. These solutions are given by a sum of hypergeometric functions, generalizing the results obtained in the paper "Black-Scholes Goes Hypergeometric" 2. For two-dimensional processes, more precisely stochastic volatility models, the classification is achieved for a specific class called gauge-free models including the Heston model, the 3/2-model and the geometric Brownian model. We then present a new exact stochastic volatility model belonging to this class.
\end{abstract}

\section{INTRODUCTION}

For most mathematical models of asset dynamics, an exact solution for the corresponding Kolmogorov \& Black-Scholes equation is usually not available: there are, however, a few notable exceptions. The known solutions for local volatility models are the constant elastic of variance (CEV) 9] including the classical log-normal Black-Scholes process. For the instantaneous short rate models, there are the CIR process [10] (Bessel process) and the Vacicek-Hull-White process 13] (Ornstein-Uhlenbeck process). For stochastic volatility models, the known exact solutions are the Heston model [12, the 3/2-model and the geometric Brownian model [18. These analytical solutions can be used for calibrating a model quickly and efficiently or can serve as a benchmark for testing the implementation of more realistic models requiring intensive numerical computation (Monte-Carlo, partial differential equation). For example, the existence of a closed-form solution for the price of a vanilla option in the Heston model allows us to quickly calibrate the model to the implied volatilities observed on the market. The calibrated model can then be used to value path-dependent exotic options using, for example, a Monte-Carlo methodology.

In this paper, we show how to obtain new analytic solutions to the Kolmogorov \& Black-Scholes equation, which we refer to as KBS throughout the rest of the paper, for $1 \mathrm{~d} \& 2 \mathrm{~d}$ diffusion processes. In order to get to our classification, we first present a general reduction method to simplify the multi-dimensional KBS equation. Rewriting the KBS equation as a heat kernel equation on a Riemannian manifold endowed with an Abelian connection, we show that this covariant equation can be simplified using both the group of diffeomorphisms (i.e. change of variables) and the group of Abelian gauge transformations. In particular for the models admitting a flat Abelian connection,

Key words and phrases. Solvable diffusion process, supersymmetry, differential geometry. 
PIERRE HENRY-LABORDÈRE

there always exists a gauge transformation that eliminates the Abelian connection of the diffusion operator.

In the second part we apply the reduction method, previously presented, to a one-dimensional, time-homogeneous diffusion process. Modulo a change of variable, the metric becomes flat and the Abelian connection is an exact one-form for which a gauge transformation can always be applied. Using these two transformations, the resulting KBS equation becomes a Euclidean Schrodinger equation with a scalar potential. Extensive work has already been done to classify the set of scalar potentials which admit an exact solution. In particular, using a supersymmetric formulation of the Schrodinger equation which consists in doubling the BKS equation with another equation, we show how to generate a hierarchy of new solvable diffusion processes starting from a solvable diffusion process (for e.g. a Natanzon potential [22]). In this context, the local volatility function is identified with a superpotential. Applying supersymmetric transformations on the Natanzon potential (which is the most general potential for which the Schrodinger equation can be reduced to either a hypergeometric or a confluent equation), we obtain a new class of solvable one-dimensional diffusion processes which are characterized by six parameters.

The classification of one-dimensional time-homogeneous solvable diffusion processes for which the solution to the KBS equation can be written as a hypergeometric function has been achieved in [2, 3, 4, 5, 16] using the well known Natanzon classification. The application of supersymmetric techniques to the classification of solvable potentials for the Schrodinger equation has been reviewed in [8] where a large number of references can be found. For the Kolmogorov \& Fokker-Planck equations, one can consult [17, 15].

In the last part we pursue this classification for stochastic volatility models which admit a flat Abelian connection: we refer to these as gauge-free models. Surprisingly, this class includes all the well known exact stochastic volatility models (i.e. the Heston model, the 3/2-model and the geometric Brownian model). For this gauge-free models, we reduce the two-dimensional KBS equation to a Euclidean Schrodinger equation with a scalar potential. Then, we present a new exact stochastic volatility model which is a combination of the Heston and 3/2-models.

\section{Reduction Method}

In this section, we explain how to simplify the KBS equation. This reduction method will be used in the next section to classify the solvable one and two dimensional time-homogeneous processes. This method is already well known for a one-dimensional process and is presented in [7, 19, 20, for example. However, the extension of this method to multi-dimensional diffusion processes requires the introduction of differential geometric objects such as a metric and an Abelian connection on a Riemannian manifold, as we will presently explain.

Let us assume that our time-homogeneous multi-dimensional diffusion model depends on $n$ random processes $x_{i}$ which can be either traded assets or hidden Markov processes (such as a stochastic volatility $a$ or an instantaneous short rate $r$ ). Let us denote $x=\left(x_{i}\right)_{i=1, \cdots, n}$, with initial conditions $\alpha=\left(\alpha_{i}\right)_{i=1, \cdots, n}$. These variables $x_{i}$ satisfy the following stochastic differential equations (SDE)

$$
\begin{aligned}
& d x^{i}=b^{i}(x) d t+\sigma^{i}(x) d W_{i} \\
& d W_{i} d W_{j}=\rho_{i j}(x) d t
\end{aligned}
$$

with the initial condition $x_{i}(t=0)=\alpha_{i}$. The no-arbitrage condition implies that there exists an equivalent measure $\mathbb{P}$ such that the traded assets are (local) martingales under this measure. For $\mathbb{P}$, the drifts $b_{i}$ are consequently zero for the traded assets. Note that the measure $\mathbb{P}$ is not unique 
as the market is not necessarily complete. Finally, the fair value of a (European) option, with payoff $f\left(x^{i}\right)$ at maturity $T$, is given by the discounted mean value of the payoff $f$ conditional on the filtration $\mathcal{F}_{t}$ generated by the Brownian motions $\left\{W_{s \leq t}^{i}\right\}$

$$
\mathcal{C}(\alpha, t, T)=\mathbb{E}^{\mathbb{P}}\left[e^{-\int_{t}^{T} r_{s} d s} f \mid \mathcal{F}_{t}\right]
$$

with $r_{s}$ the instantaneous short rate. This mean-value depends on the probability density $p\left(x_{i}, T \mid \alpha, t\right)$ which satisfies the backward Kolmogorov equation $\left(\tau=T-t, \partial_{i}=\frac{\partial}{\partial x_{i}}\right)$

$$
\frac{\partial p}{\partial \tau}=b^{i} \partial_{i} p+\frac{1}{2} \rho_{i j} \sigma^{i} \sigma^{j} \partial_{i j} p
$$

with initial condition $p(\tau=0)=\delta(x-\alpha)$. In this equation we have used the Einstein convention meaning that two repeated indices are summed.

Using the Feymann-Kac theorem, one can show that the fair value $\mathcal{C}$ for the option satisfies the Black-Scholes equation $\left(\partial_{i}=\frac{\partial}{\partial \alpha_{i}}\right)$

$$
\frac{\partial \mathcal{C}}{\partial \tau}=b^{i} \partial_{i} \mathcal{C}+g^{i j} \partial_{i j} \mathcal{C}-r(\alpha) \mathcal{C}
$$

with initial condition $\mathcal{C}(\tau=0, \alpha)=f(\alpha)$.

In the following, the partial differential equations (PDE) 2.42.5) will be interpreted as the heat kernel on a general smooth $n$-dimensional manifold $M$ (without a boundary) endowed with a metric $g_{i j}$. The inverse of the metric $g^{i j}$ is defined by

$$
g^{i j}=\frac{1}{2} \rho_{i j} \sigma^{i} \sigma^{j}
$$

and the metric ( $\rho^{i j}$ inverse of $\rho_{i j}$ )

$$
g_{i j}=2 \frac{\rho^{i j}}{\sigma^{i} \sigma^{j}}
$$

The differential operator

$$
D=b^{i} \partial_{i}+g^{i j} \partial_{i j}
$$

which appears in (2.4) is a second order elliptic operator of Laplace type. We can then show that there is a unique connection $\nabla$ on $\mathcal{L}$, a line bundle over $M$, and a unique smooth function $Q$ on $M$ such that

$$
\begin{aligned}
D & \equiv g^{i j} \nabla_{i} \nabla_{j}+Q \\
& =g^{-\frac{1}{2}}\left(\partial_{i}+\mathcal{A}_{i}\right) g^{\frac{1}{2}} g^{i j}\left(\partial_{j}+\mathcal{A}_{j}\right)+Q
\end{aligned}
$$

with $g=\operatorname{det}\left[g_{i j}\right]$. We may express the connection $\mathcal{A}^{i}$ and $Q$ as a function of the drift $b_{i}$ and the metric $g_{i j}$ by identifying in (2.10) the terms $\partial_{i}$ and $\partial_{i j}$ with those in (2.8). We find

$$
\begin{aligned}
\mathcal{A}^{i} & =\frac{1}{2}\left(b^{i}-g^{-\frac{1}{2}} \partial_{j}\left(g^{1 / 2} g^{i j}\right)\right) \\
Q & =g^{i j}\left(\mathcal{A}_{i} \mathcal{A}_{j}-b_{j} \mathcal{A}_{i}-\partial_{j} \mathcal{A}_{i}\right)
\end{aligned}
$$


Note that the Latin indices $i, j \cdots$ can be lowered or raised using the metric $g_{i j}$ or its inverse $g^{i j}$. For example $\mathcal{A}_{i}=g_{i j} \mathcal{A}^{j}=\frac{1}{2}\left(g_{i k} b^{k}-\frac{1}{2} \partial_{i} \ln (g)-g_{i p} \partial_{k} g^{p k}\right)$.

Using this connection, 2.4 can be rewritten in the covariant way, i.e.

$$
\frac{\partial}{\partial \tau} p(x, \alpha, \tau)=D p(x, \alpha, \tau)
$$

If we take $\mathcal{A}_{i}=0, Q=0$ then $D$ becomes the Laplace-Beltrami operator (or Laplacian) $\Delta=$ $g^{-\frac{1}{2}} \partial_{i} g^{\frac{1}{2}} g^{i j} \partial_{j}$. For this configuration, 2.13) will be called the Laplacian heat kernel. Similarly, the Black-Scholes equation 2.5 can be rewritten

$$
\frac{\partial}{\partial \tau} \mathcal{C}(\alpha, \tau)=(D-r) \mathcal{C}(\alpha, \tau)
$$

The Heat kernel equation can now be simplified by applying the actions of the following groups.

$\triangleright$ The group of diffeomorphisms $\operatorname{Diff}(\mathcal{M})$ which acts on the metric $g_{i j}$ and the connection $\mathcal{A}_{i}$ by

$$
\begin{aligned}
f^{*} g_{i j} & =g_{p k} \partial_{i} f^{p}(x) \partial_{j} f^{k}(x) \\
f^{*} \mathcal{A}_{i} & =\mathcal{A}_{p} \partial_{i} f^{p}(x), f \in \operatorname{Diff}(\mathcal{M})
\end{aligned}
$$

$\triangleright$ The group of gauge transformations $\mathcal{G}$ which acts on the conditional probability (and the fair value $\mathcal{C}$ ) by

$$
\begin{aligned}
p^{\prime} & =e^{\chi(x, \tau)-\chi(x=\alpha, \tau=0)} p \\
\mathcal{C}^{\prime} & =e^{\chi(\alpha, \tau)} \mathcal{C}
\end{aligned}
$$

Then $p^{\prime}\left(\mathcal{C}^{\prime}\right)$ satisfies the same equation as $p(\mathcal{C})$ (2.13) only with

$$
\begin{aligned}
\mathcal{A}_{i}^{\prime} & \equiv \mathcal{A}_{i}-\partial_{i} \chi \\
Q^{\prime} & \equiv Q+\partial_{\tau} \chi
\end{aligned}
$$

The constant phase $e^{\chi(x=\alpha, \tau=0)}$ has been added so that $p$ and $p^{\prime}$ satisfy the same boundary condition at $\tau=0$. The above transformation is called a gauge transformation. If the connection $\mathcal{A}$ is an exact form (meaning that there exists a smooth function $\Lambda$ such that $\mathcal{A}_{i}=\partial_{i} \Lambda$ ), then by applying a gauge transformation, we can eliminate the connection so that the heat kernel equation for $p^{\prime}$ (or $\mathcal{C}^{\prime}$ ) has a connection equal to zero. It can be shown that for a simply-connected manifold, the statement " $\mathcal{A}$ is exact" is equivalent to $\mathcal{F}=0$, where $\mathcal{F}$ is the 2 -form curvature given in a specific coordinate system by

$$
\mathcal{F}_{i j}=\partial_{i} \mathcal{A}_{j}-\partial_{j} \mathcal{A}_{i}
$$

In the following, we will restrict our classification to those processes for which $\mathcal{F}=0$, meaning there exists a gauge transformation such that the transformed connection vanishes. The operator $D$ reduces in this case to the symmetric operator $D=\Delta+Q$ for which we can use an eigenvector expansion.

\section{Spectral decomposition}


This spectral decomposition is valid only if the symmetric operator $D$ is an (unbounded) selfadjoint operator or admits a self-adjoint extension. This will depend strongly on the boundary conditions. In order to show that $D$ is self-adjoint or admits self-adjoint extensions, we can use the deficiency indices technique introduced by Von Neumann ${ }^{1}$ ([23] and see [6] for a pedagogical introduction).

After proving that $D$ is a self-adjoint operator or admits a self-adjoint extension, the conditional probability (or the fair value) can then be expanded over a complete basis of orthonormal eigenvectors $\phi_{n}(x)$

$$
p\left(x, \tau, x_{0}\right)=\sum_{n} e^{-E_{n} \tau} \phi_{n}(x) \phi_{n}\left(x_{0}\right)
$$

with $D \phi_{n}(x)=E_{n} \phi_{n}(x)$. The discrete summation over $n$ can also include a continuous summation according to a specific measure $\mu(E)$ if the spectrum contains a continuous part.

\section{1D Time-Homogeneous Diffusion MOdeLS}

In the next section, we apply the general reduction method, presented previously, to the onedimensional KBS equation. Similar reduction to a Schrodinger equation with a scalar potential (without any references to differential geometry) can be found in [7, 19, 20. We then find the supersymmetric partner of this Schrodinger equation and show how to generate new exact solutions (for Vanilla options).

3.1. Reduction method. Consider a one-dimensional, time-homogeneous diffusion process with drift $^{2}$

$$
d f=\mu(f) d t+\sigma(f) d W
$$

\footnotetext{
${ }^{1}$ Before explaining this technique, let us recall some definitions. An operator $(H, \mathcal{D}(H))$ defined on a Hilbert space $\mathcal{H}$ is said to be densely defined if the subset $\mathcal{D}(H)$ is dense in $\mathcal{H}$, i.e. for any $\phi \in \mathcal{H}$ one can find in $\mathcal{D}(H)$ a sequence which converges in norm to $\phi$.

The domain $\mathcal{D}\left(H^{\dagger}\right)$ of an adjoint operator of an (unbounded) operator $H$ with dense domain $\mathcal{D}$ is the space of functions $\psi$ such that the linear form $\phi \rightarrow(\psi, H \phi)$ is continuous for the norm of $\mathcal{H}$. Hence using Riesz' theorem, there exists a unique $\phi^{\prime}$ such that $(\psi, H \phi)=\left(\psi^{\prime}, \phi\right)$ with $(.,$.$) the scalar product on the Hilbert space. By definition,$ we set $H^{\dagger} \psi=\psi^{\prime}$.

An operator $H$ is called symmetric if for all $\phi, \psi \in \mathcal{D}(H)$, we have $(H \phi, \psi)=(\psi, H \psi) . H$ is self-adjoint if additionally $\mathcal{D}\left(H^{\dagger}\right)=\mathcal{D}(H)$. Let us assume that $(H, \mathcal{D}(H))$ is densely defined, symmetric and closed with adjoint $\left(H^{\dagger}, \mathcal{D}\left(H^{\dagger}\right)\right)$. The deficiency indices are then defined by

$$
n_{ \pm}=\operatorname{Ker}\left(H^{\dagger} \mp i I d\right)
$$

Theorem 2.0.1. For an operator with deficiency indices $\left(n_{-}, n_{+}\right)$, there are three possibilities:

(1) If $n_{-}=n_{+}=0$, then $H$ is self-adjoint (necessary and sufficient condition)

(2) If $n_{+}=n_{-}=n \geq 1$, then $H$ has infinitely many self-adjoint extensions, parameterized by the unitary group $U(n)$.

(3) If $n_{-} \neq n_{+}$, then $H$ has no self-adjoint extension.

Note that if the deficiency indices are given by $(n, n)$ then the spectrum is discrete. Moreover, if $H$ admits a self-adjoint extension, the resulting conditional probability is not unique but depends on the boundary conditions which are parameterized by the unitary group $U(n)$.

${ }^{2}$ The time-dependent process $d f=\mu(f) A^{2}(t) d t+\sigma(f) A(t) d W$ is equivalent to this process under the change of local time $t^{\prime}=\int^{t} A^{2}(s) d s$
} 
This process has been used as the basis for various mathematical models in finance. If $f$ is a traded asset (i.e. a forward), the drift vanishes in the forward measure and we have a local volatility model where we assume that the volatility is only a function of $f$. The one-dimensional process is not necessarily driftless as the random variable is not a traded asset as it is the case for an instantaneous short rate model, or a model of stochastic volatility.

In our framework, this process corresponds to a (one-dimensional) real curve endowed with the metric $g_{f f}=\frac{2}{\sigma(f)^{2}}$. For the new coordinate

$$
s(f)=\sqrt{2} \int_{f_{0}}^{f} \frac{d f^{\prime}}{\sigma\left(f^{\prime}\right)}
$$

the metric is flat: $g_{s s}=1$. The Laplace-Beltrami operator therefore becomes $\Delta=\partial_{s}^{2}$.

Using the definition (2.11), 2.12), we find that the Abelian connection $\mathcal{A}_{f}$ and the function $Q$ are given by

$$
\begin{aligned}
& \mathcal{A}_{f}=-\frac{1}{2} \partial_{f} \ln (\sigma(f))+\frac{\mu(f)}{\sigma^{2}(f)} \\
& Q=\frac{1}{4}\left(\sigma(f) \sigma^{\prime \prime}(f)-\frac{1}{2} \sigma^{\prime}(f)^{2}\right)-\frac{\mu^{\prime}(f)}{2}+\frac{\mu(f) \sigma^{\prime}(f)}{\sigma(f)}-\frac{\mu(f)}{2 \sigma^{2}(f)}
\end{aligned}
$$

In this case, by applying a gauge transformation on the conditional probability $p, P=\frac{\sigma\left(f_{0}\right)}{\sqrt{2}} e^{\Lambda} p$ with

$$
\Lambda=-\frac{1}{2} \ln \left(\frac{\sigma(f)}{\sigma\left(f_{0}\right)}\right)+\int_{f_{0}}^{f} \frac{\mu\left(f^{\prime}\right)}{\sigma^{2}\left(f^{\prime}\right)} d f^{\prime}
$$

then the connection vanishes and $P$ satisfies a heat kernel with a scalar potential $Q(s)$ (in the $s$ flat coordinate)

$$
\partial_{\tau} P(s, \tau)=\left(\partial_{s}^{2}+Q(s)\right) P(s, \tau)
$$

The solution $P$ has been scaled by the (constant) factor $\frac{\sigma\left(f_{0}\right)}{\sqrt{2}}$ in order to have the initial condition $\lim _{\tau \rightarrow 0} P(s, \tau)=\delta(s)$. Moreover, $Q$ is given in the $s$ coordinate by

$$
Q=\frac{1}{2}(\ln (\sigma))^{\prime \prime}(s)-\frac{1}{4}\left((\ln (\sigma))^{\prime}(s)\right)^{2}-\frac{\mu^{\prime}(s)}{\sqrt{2} \sigma(s)}+\frac{\sqrt{2} \mu(s) \sigma^{\prime}(s)}{\sigma(s)^{2}}-\frac{\mu^{2}(s)}{2 \sigma^{2}(s)}
$$

where the prime' indicates a derivative according to $s$.

Example 3.1.1 (quadratic volatility process). Let us assume that $f$ satisfies a driftless process (i.e. $\mu(f)=0)$. The Black-Scholes equation reduces to the heat kernel on $\mathbb{R}$ if $Q(s)=$ constant (i.e. $Q(s)$ is zero modulo a time-dependent gauge transformation) which is equivalent to $\sigma(f)=\alpha f^{2}+\beta f+\gamma$ (i.e. the quadratic volatility model, also called the hyperbolic model [19]).

Example 3.1.2 (CEV process). For the CEV process $d f=f^{\beta} d W_{t} \mu(f)=0$, the potential is $Q(s)=\frac{\beta(\beta-2)}{4(1-\beta)^{2} s^{2}}$ for $\beta \neq 1$ and $Q(s)=-\frac{1}{8}$ for $\beta=1$. 
If the Hamiltonian $D=\left(-\partial_{s}^{2}-Q\right)$ is self-adjoint or admits self-adjoint extensions, the spectral decomposition can be used and the conditional probability can be decomposed using a complete basis of orthonormal eigenvectors $\phi_{n}(s)$.

$$
P(s, \tau)=\sum_{n} \phi_{n}(s) \phi_{n}^{\dagger}\left(s_{0}\right) e^{-E_{n} \tau}
$$

with $\phi_{n}(s)$ satisfying

$$
D \phi_{n}(s)=E_{n} \phi_{n}(s)
$$

\section{Boundary conditions}

For a one-dimensional diffusion process, one don't need to use the deficiency indices technique as the complete classification of the boundary conditions is given by Feller's classification. More precisely, for a 1D time-homogeneous diffusion process, the boundary falls into one of the four following types: regular, entrance, exit or natural. For entrance, exit or natural, no boundary conditions are needed. For a regular boundary, the conditional probability is not unique but depends on the boundary conditions. It corresponds to the case when the deficiency indices are $(n, n)$. The boundary classification depends on the behavior of the following functions $S(c, d)=\int_{c}^{d} s(f) d f$, $M(c, d)=\int_{c}^{d} m(f) d f, \Sigma(e)=\lim _{c \rightarrow e} \int_{c}^{d} S(c, f) m(f) d f$ and $N(e)=\lim _{c \rightarrow e} \int_{c}^{d} S(x, d) m(x) d x$ with $s(f)=e^{-2 \int^{f} \frac{\mu(x) d x}{\sigma(x)^{2}}}$ and $m(f)=\frac{1}{\sigma^{2}(f) s(f)}$ (see Table 1 below).

\begin{tabular}{|c|cccc|}
\hline Boundary type & $S(e, d)$ & $M(e, d)$ & $\Sigma(e)$ & $N(e)$ \\
\hline Regular & $<\infty$ & $<\infty$ & $<\infty$ & $<\infty$ \\
\hline Exit & $<\infty$ & $=\infty$ & $<\infty$ & $<\infty$ \\
\hline Entrance & $=\infty$ & $<\infty$ & $<\infty$ & $<\infty$ \\
\hline Natural & $<\infty$ & $=\infty$ & $=\infty$ & $=\infty$ \\
& $=\infty$ & $<\infty$ & $=\infty$ & $=\infty$ \\
& $=\infty$ & $=\infty$ & $=\infty$ & $=\infty$ \\
\hline
\end{tabular}

TABLE 1. Feller's Classification.

Example 3.1.3 (Vanilla option with constant interest rate). We have that the forward $f$ satisfies a driftless process (i.e. $\mu(f)=0$ ). The value at $t$ of a European option (with strike $K$ and expiry date $T)$ is then given by $(\tau=T-t)$

$$
\mathcal{C}\left(f_{t}, K, \tau\right)=e^{-r \tau} \int_{K}^{\infty}(f-K) p\left(f, \tau \mid f_{t}\right) d f
$$

Doing an integration by parts, or equivalently, applying the Tanaka-Meyer formula on the payoff $\left(S_{t}-K\right)^{+} \mathbb{4}$ we can show that the vanilla option $\mathcal{C}$ can be rewritten as

$$
\mathcal{C}\left(f_{t}, K, \tau\right)=e^{-r \tau}\left(f_{t}-K\right)^{+}+e^{-r \tau} \frac{\sigma^{2}(K)}{2} \int_{0}^{\tau} d t^{\prime} p\left(K, t^{\prime} \mid f_{t}\right)
$$


Using the relation between the conditional probability $p\left(f, t^{\prime} \mid f_{t}\right)$ and its gauge-transform $P\left(s(f), t^{\prime} \mid s_{t}\right)$, we obtain

$$
\mathcal{C}\left(f_{t}, K, \tau\right)=e^{-r \tau}\left(\left(f_{t}-K\right)^{+}+\frac{\sigma(K)^{\frac{5}{2}}}{\sqrt{2} \sigma\left(f_{t}\right)^{\frac{3}{2}}} \int_{0}^{\tau} P\left(s(K), t^{\prime} \mid s_{t}\right) d t^{\prime}\right)
$$

Plugging the expression for $P\left(s, t^{\prime} \mid s_{t}\right)$ (3.8) into (3.12) and doing the integration over the time $t$, we obtain 4

$$
\mathcal{C}\left(f_{t}, K, \tau\right)=e^{-r \tau}\left(\left(f_{t}-K\right)^{+}+\frac{\sigma(K)^{\frac{5}{2}}}{\sqrt{2} \sigma\left(f_{t}\right)^{\frac{3}{2}}} \sum_{n} \phi_{n}(s(K)) \phi_{n}\left(s_{t}\right) \frac{\left(1-e^{-E_{n} \tau}\right)}{E_{n}}\right)
$$

A specific local volatility model will give an exact solution for a vanilla option if we can find the eigenvalues and eigenvectors for the corresponding Euclidean Schrodinger equation with a scalar potential. As examples of solvable potentials, one can cite the harmonic oscillator, Coulomb, Morse, Poschl-Teller I\&II, Eckart and Manning-Rosen potentials. The classification of solvable scalar potentials was initiated by Natanzon [22]. This work provides the most general potential for which the Schrodinger equation can be reduced to either a hypergeometric or confluent equation. We will review in the following section the Natanzon potential, which depends on 6 parameters. We will later show that the Schrodinger equation can be doubled into a set of two independent Schrodinger equations with two different scalar potentials which transform into each other under a supersymmetric transformation. Moreover, if one scalar potential is solvable, the other one is. Applying this technique to the Natanzon potential, we will obtain a new class of solvable potentials corresponding to a new class of solvable diffusion processes. For these models, the solution to the KBS equation is given by a sum of hypergeometric functions.

3.2. Solvable (super)potentials. In this section, we show that the Schrodinger equation can be formulated using supersymmetric techniques (see [8] for a nice review). In particular, the local volatility will be identified as a superpotential. Using this formalism, we show how to generate a hierarchy of solvable diffusion models starting from a known solvable superpotential, for example the hypergeometric or confluent hypergeometric Natanzon superpotential.

3.2.1. Superpotential and local volatility. Let us write the Kolmogorov equation (3.9) in the following way by introducing the first-order operator $A_{1}$ and its formal adjoint $A_{1}^{\dagger} 3$

$$
E_{n}^{(1)} \phi_{n}^{(1)}=A_{1}^{\dagger} A_{1} \phi_{n}^{(1)}
$$

with $A_{1}=\partial_{s}+W^{(1)}(s), A_{1}^{\dagger}=-\partial_{s}+W^{(1)}(s)$. $W^{(1)}$ is called the superpotential which satisfies the Riccatti equation

$$
Q^{(1)}(s)=\partial_{s} W^{(1)}(s)-W^{(1)^{2}}(s)
$$

Surprisingly, this equation is trivially solved for our specific expression for $Q^{(1)}$ (3.7) (even with a drift $\mu(f) !)$

\footnotetext{
${ }^{3}$ In order to obtain the correct adjoint operator on $\mathbb{R}^{+}$, we impose the absorbing boundary condition $\phi_{n}(s=$ $0)=0$
} 


$$
W^{(1)}(s)=\frac{1}{2} \frac{d \ln \sigma^{(1)}(s)}{d s}-\frac{\mu^{(1)}(s)}{\sqrt{2} \sigma^{(1)}(s)}
$$

In particular, for zero drift, the local volatility function is directly related to the superpotential by $\sigma(s)=e^{2 \int^{s} W(z) d z}$. A similar correspondence between the superpotential and driftless diffusion process has been found in [17] [15]. Moreover, if we have a family of solvable superpotentials $W_{\text {solvable }}^{(1)}(s)$, we can always find an analytic solution to the Kolmogorov equation for any diffusion term $\sigma^{(1)}(s)$ by adjusting the drift with the relation 3.3.

$$
\mu^{(1)}(s)=\frac{\sigma^{(1)^{\prime}}(s)}{\sqrt{2}}-\sqrt{2} \sigma^{(1)}(s) W_{\text {solvable }}^{(1)}(s)
$$

Note that (3.1) admits a zero eigenvalue if and only if the Kolmogorov equation admits a stationary distribution. By observing that $A_{1}^{\dagger} A_{1} \phi_{0}^{(1)}=0$ is equivalent to $A_{1} \phi_{0}^{(1)}=0$, we obtain the stationary distribution

$$
\phi_{0}^{(1)}(s)=C e^{-\int^{s} W^{(1)}(z) d z}
$$

with $C$ a normalization constant. Therefore, the stationary distribution will exist if the superpotential is normalisable (i.e. $\left.\phi_{0}^{(1)}(s) \in L^{2}\right)$.

Example 3.2.1 (Coulomb superpotential and CEV process). The CEV process corresponding to $\sigma(f)=\sigma_{0} f^{\beta}$ and $\mu(f)=0$ has the Coulomb superpotential $W(s)=\frac{\beta}{2(1-\beta) s}$.

Next, we define the Scholes-Black equation by intervening the operator $A_{1}$ and $A_{1}^{\dagger}$

$$
\begin{aligned}
E_{n}^{(2)} \phi_{n}^{(2)}(s) & =A_{1} A_{1}^{\dagger} \phi_{n}^{(2)}(s) \\
& =\left(-\partial_{s}^{2}-Q^{(2)}(s)\right) \phi_{n}^{(2)}(s)
\end{aligned}
$$

We obtain a new Schrodinger equation with the partner potential

$$
Q^{(2)}(s)=-\partial_{s} W^{(1)}-\left(W^{(1)}\right)^{2}
$$

Plugging our expression for the superpotential (3.3) in (3.8), we have

$$
\left.Q^{(2)}=-\frac{1}{2}\left(\ln \left(\sigma^{(1)}\right)\right)^{\prime \prime}(s)-\frac{1}{4}\left(\ln \left(\sigma^{(1)}\right)\right)^{\prime}(s)\right)^{2}+\frac{\mu^{(1)^{\prime}}(s)}{\sqrt{2} \sigma^{(1)}(s)}-\frac{\mu^{(1)^{2}}(s)}{2 \sigma^{(1)^{2}}(s)}
$$

In the same way as before, $H_{2}$ admits a zero eigenvector (i.e. stationary distribution) if $\phi_{0}^{(2)}(s)=$ $C e^{\int^{s} W^{(1)}(z) d z}$ is normalisable.

Remark 3.2.2. In physics, the supersymmetry (SUSY) is said to be broken if at least one of the eigenvectors $\phi_{0}^{(1,2)}(s)$ exists. Otherwise, SUSY is said to be broken dynamically. 
Now we want to show that if we can solve the equation (3.1) then we have automatically a solution to (3.7) and vice-versa. The SUSY-partner Hamiltonians $H_{1}=A_{1}^{\dagger} A_{1}$ and $H_{2}=A_{1} A_{1}^{\dagger}$ obey the relations $A_{1}^{\dagger} H_{2}=H_{1} A_{1}^{\dagger}$ and $H_{2} A_{1}=A_{1} H_{1}$. As a consequence $H_{1}$ and $H_{2}$ are isospectral. More precisely, the strictly positive eigenvalues all coincide and the corresponding eigenvectors are related by the supercharge operators $A_{1}$ and $A_{1}^{\dagger}$ :

$\triangleright$ If $H_{1}$ admits a zero eigenvalue (i.e. broken supersymmetry), we have the relation

$$
\begin{aligned}
& E_{n}^{(2)}=E_{n+1}^{(1)} ; E_{0}^{(1)}=0 ; \phi_{0}^{(1)}(s)=C e^{-\int^{s} W^{(1)}(z) d z} \\
& \phi_{n}^{(2)}(s)=\left(E_{n+1}^{(1)}\right)^{-\frac{1}{2}} A_{1} \phi_{n+1}^{(1)}(s) \\
& \phi_{n}^{(1)}(s)=\left(E_{n}^{2}\right)^{-\frac{1}{2}} A_{1}^{\dagger} \phi_{n}^{(2)}(s)
\end{aligned}
$$

$\triangleright$ If $H_{1}$ (and $H_{2}$ ) doesn't admit a zero eigenvalue (i.e. unbroken supersymmetry)

$$
\begin{aligned}
& E_{n}^{(2)}=E_{n}^{(1)} \\
& \phi_{n}^{(2)}(s)=\left(E_{n}^{(1)}\right)^{-\frac{1}{2}} A_{1} \phi_{n}^{(1)}(s) \\
& \phi_{n}^{(1)}(s)=\left(E_{n}^{2}\right)^{-\frac{1}{2}} A_{1}^{\dagger} \phi_{n}^{(2)}(s)
\end{aligned}
$$

In the broken SUSY case, there are no zero modes and consequently the spectrum of $H_{1}$ and $H_{2}$ are the same. One can then obtain the solution to the Scholes-Black (resp. Black-Scholes) equation if the eigenvalues/eigenvectors of the Black-Scholes (resp. Scholes-Black) are known. We clarify this correspondence by studying a specific example: the CEV process $d f=f^{\beta} d W$. In particular, we show that for $\beta=\frac{2}{3}$, the partner superpotential vanishes. It is therefore simpler to solve the Scholes-Black equation as Scholes-Black (rather than Black-Scholes) reduces to the heat kernel on $\mathbb{R}^{+}$. Applying a supersymmetric transformation on the Scholes-Black equation, we can then derive the solution to the Black-Scholes equation.

Example 3.2.3 (CEV with $\beta=2 / 3$ and Bachelier process). We saw previously that the superpotential associated with the CEV process is given by

$$
W^{(1)}(s)=\frac{\beta}{2 s(1-\beta)}
$$

with the flat coordinate $s=\frac{\sqrt{2} f^{1-\beta}}{(1-\beta)}$ and the potential 3.7]

$$
Q^{(1)}(s)=\frac{\beta(\beta-2)}{4(1-\beta)^{2} s^{2}}
$$

from which we deduce the partner potential (3.9)

$$
Q^{(2)}(s)=\frac{\beta(2-3 \beta)}{4(1-\beta)^{2} s^{2}}
$$

This partner potential corresponds to the potential of a CEV process $d f=f^{B} d W$ with $B$ given as a function of $\beta$ by 


$$
\frac{B(B-2)}{(1-B)^{2}}=\frac{\beta(2-3 \beta)}{(1-\beta)^{2}}
$$

Surprisingly, we observe that for $\beta=\frac{2}{3}, Q^{(2)}(s)$ cancels and the corresponding partner local volatility model is the Bachelier model $d f=d W$ for which the heat kernel is given by the normal distribution. The eigenvectors of the supersymmetric Hamiltonian partner $H_{2}=-\partial_{s}^{2}$ to $H_{1}$ are given by (with the absorbing boundary condition $\phi_{n}(0)=0$ )

$$
\phi^{(2)}(s, E)=\frac{\sin (\sqrt{E} s)}{\sqrt{4 \pi} E^{\frac{1}{4}}}
$$

with a continuous spectrum $\mathbb{R}^{+}$. Applying the supersymmetric transformation (3.12), we obtain the eigenvectors for the Hamiltonian $H_{1}=-\partial_{s}^{2}+\frac{2}{s^{2}}$ corresponding to the CEV process with $\beta=\frac{2}{3}$

$$
\begin{aligned}
\phi^{(1)}(s, E) & =E^{-\frac{1}{2}}\left(-\partial_{s}+\frac{1}{s}\right) \phi^{(2)}(s, E) \\
& =\frac{1}{\sqrt{4 \pi} E^{\frac{3}{4}}}\left(-\sqrt{E} \cos (\sqrt{E} s)+\frac{\sin (\sqrt{E} s)}{s}\right)
\end{aligned}
$$

Plugging this expression in (3.13), we obtain the fair value for a vanilla option which can be integrated and written in terms of the cumulative distribution [19]

$$
e^{r \tau} \mathcal{C}\left(f_{t}, K, \tau\right)=\left(f_{t}-K\right)^{+}+\frac{K^{\frac{5}{3}}}{\sqrt{2} f_{t}} \int_{0}^{\infty} d E \frac{\left(1-e^{-E \tau}\right)}{E} \phi^{(2)}(s, E) \phi^{(2)}\left(s_{0}, E\right)
$$

The fact that the CEV model with $\beta=\frac{2}{3}$ depends on the normal cumulative distribution and is therefore related to the heat kernel on $\mathbb{R}^{+}$has been observed empirically by [19. Here we have seen that it corresponds to the fact that the supersymmetric partner potential vanishes for this particular value of $\beta$.

3.3. Hierarchy of solvable diffusion processes. In the previous section we saw that the operators $A_{1}$ and $A_{1}^{\dagger}$ can be used to factorize the Hamiltonian $H_{1}$. These operators depend on the superpotential $W^{(1)}$ which is determined once we know the first eigenvector $\phi_{0}^{(1)}(s)$ of $H_{1}$ (3.5.). We have assumed that $H_{1}$ admits a zero eigenvalue. By shifting the energy $E$ it is always possible to achieve this condition. The partner Schrodinger equation (3.7) can then be recast into a Schrodinger equation with a zero eigenvalue

$$
H_{(2)}=A_{1} A_{1}^{\dagger}=A_{2}^{\dagger} A_{2}+E_{1}^{(1)}
$$

where $A_{2} \equiv \partial_{s}+W_{2}(s)$ and $A_{2}^{\dagger} \equiv-\partial_{s}+W_{2}(s)$,

$$
W^{(2)}(s) \equiv \frac{1}{2} \frac{d \ln \sigma^{(2)}(s)}{d s}-\frac{\mu^{(2)}(s)}{\sqrt{2} \sigma^{(2)}(s)}
$$


We have introduced the notation that in $E_{n}^{(m)}, n$ denotes the energy level and $(m)$ refers to the $\mathrm{m}^{\text {th }}$ Hamiltonian $H_{m}$. By construction, this new Hamiltonian $H_{2}=A_{2}^{\dagger} A_{2}+E_{1}^{(1)}$ is solvable as $A_{1} A_{1}^{\dagger}$ is and the associated diffusion process with volatility $\sigma^{(2)}$ and drift $\mu^{(2)}$ satisfy (3.2). The superpotential $W^{(2)}(s)$ is determined by the first eigenvector of $H_{2}, \phi_{0}^{(2)}(s)$,

$$
W^{(2)}(s)=-\frac{d \ln \left(\phi_{0}^{(2)}\right)(s)}{d s}
$$

We can then apply a supersymmetric transformation on $H_{2}$. The new Hamiltonian $H_{3}$ can be refactorised exactly in the same way we did for $H_{2}$. Finally, it is not difficult to see that if $H_{1}$ admits $p$ discrete (normalisable) eigenvectors, then one can generate a family of solvable Hamiltonians $H_{m}$ (with a zero-eigenvalue by construction)

$$
H_{m}=A_{m}^{\dagger} A_{m}+E_{m-1}^{(1)}=-\partial_{s}^{2}+Q_{m}(s)
$$

where $A_{m}=\partial_{s}+W_{m}(s)$. This corresponds to the solvable diffusion process with a drift and a volatility such that

$$
W_{m}(s)=-\frac{d \ln \phi_{0}^{(m)}}{d s}=\frac{1}{2} \frac{d \ln \sigma^{(m)}(s)}{d s}-\frac{\mu^{(m)}(s)}{\sqrt{2} \sigma^{(m)}(s)}
$$

The eigenvalues/eigenvectors of $H_{m}$ are related to those of $H_{1}$ by

$$
\begin{aligned}
& E_{n}^{(m)}=E_{n+1}^{(m-1)}=\cdots=E_{n+m-1}^{(1)} \\
& \phi_{n}^{(m)}=\left(E_{n+m-1}^{(1)}-E_{m-2}^{(1)}\right)^{-\frac{1}{2}} \cdots\left(E_{n+m-1}^{(1)}-E_{0}^{(1)}\right)^{-\frac{1}{2}} A_{m-1} \cdots A_{1} \phi_{n+m-1}^{(1)}
\end{aligned}
$$

In particular, the superpotential of $H_{m}$ is determined by the $(m-1)^{\mathrm{th}}$ eigenvector of $H_{1}, \phi_{m-1}^{(1)}(s)$,

$$
\begin{aligned}
W_{m}(s) & =-\frac{d \ln \left(A_{m-1} \cdots A_{1} \phi_{m-1}^{(1)}(s)\right)}{d s} \\
& =\frac{1}{2} \frac{d \ln \sigma^{(m)}(s)}{d s}-\frac{\mu^{(m)}(s)}{\sqrt{2} \sigma^{(m)}(s)}
\end{aligned}
$$

Consequently, if we know all the $m$ discrete eigenvalues and eigenvectors of $H_{1}$, we immediately know all the energy eigenvalues and eigenfunctions of the hierarchy of $m-1$ Hamiltonians. In the following we apply this procedure starting from a known solvable superpotential, the Natanzon superpotential.

3.4. Natanzon (super)potentials. The Natanzon potential 22 is the most general potential which allows us to reduce the Schrodinger equation (3.6) to a Gauss or confluent hypergeometric equation (GHE or $\mathrm{CHE}$ ). 
3.4.1. Gauss hypergeometric potential. The potential is given by

$$
Q(s)=\frac{S(z)-1}{R(z)}-\left(\frac{r_{1}-2\left(r_{2}+r_{1}\right) z}{z(1-z)}-\frac{5}{4} \frac{\left(r_{1}^{2}-4 r_{1} r_{0}\right)}{R(z)}+r_{2}\right) \frac{z^{2}\left(1-z^{2}\right)}{R(z)^{2}}
$$

with $R(z)=r_{2} z^{2}+r_{1} z+r_{0}>0$ and $S(z)=s_{2} z^{2}+s_{1} z+s_{0}$ (two second order polynomials). The $z$ coordinate, lying in the interval $[0,1]$, is defined implicitly in terms of $s$ by the differential equation

$$
\frac{d z(s)}{d s}=\frac{2 z(1-z)}{\sqrt{R(z)}}
$$

Example 3.4.1. The hypergeometric Natanzon potential includes as special cases the Posch-Teller potential II $Q(s)=A+B \operatorname{sech}\left(\frac{2 s}{\sqrt{r_{1}}}\right)^{2}+C \operatorname{csch}\left(\frac{2 s}{\sqrt{r_{1}}}\right)^{2}$ for $r_{0}=r_{2}=0$ and the Rosen-Morse potential $Q(s)=A+B \tanh \left(\frac{2 s}{\sqrt{r_{0}}}\right)+C \operatorname{sech}\left(\frac{2 s}{\sqrt{r_{0}}}\right)^{2}, r_{1}=r_{2}=0$ with $A$ and $B$ constant.

By construction, the solution to the Schrodinger equation with a GHE potential is given in terms of the Gauss hypergeometric function $F(\alpha, \beta, \gamma, z)$

$$
\psi(s, E)=\left(z^{\prime}\right)^{-\frac{1}{2}} z^{\frac{\gamma}{2}}(1-z)^{\frac{-\gamma+\alpha+\beta+1}{2}} F(\alpha, \beta, \gamma, z)
$$

where $F(\alpha, \beta, \gamma, z)$ satisfies the differential equation 1

$$
z(1-z) \frac{d^{2} F}{d z^{2}}+(\gamma-(\alpha+\beta+1) z) \frac{d F}{d z}-\alpha \beta z=0
$$

and the most general solution to this equation (3.4) is generated by a two-dimensional vector space

$$
F(\alpha, \beta, \gamma, z)=c_{12} F_{1}(\alpha, \beta, \gamma, z)+c_{2} z^{1-\gamma} F_{1}(\alpha-\gamma+1, \beta-\gamma+1,2-\gamma, z)
$$

with ${ }_{2} F_{1}(\alpha, \beta, \gamma, z)$ satisfying ${ }_{2} F_{1}(\alpha, \beta, \gamma, 0)=1$ and $c_{1}$ and $c_{2}$ two arbitrary coefficients. The parameters $\alpha, \beta, \gamma$ depend explicitly on the eigenvalue $E$ by

$$
\begin{aligned}
1-(\alpha-\beta)^{2} & =r_{2} E+s_{2} \\
2 \gamma(\alpha+\beta-1)-4 \alpha \beta & =r_{1} E+s_{1} \\
\gamma(2-\gamma) & =r_{0} E+s_{0}
\end{aligned}
$$

In fact, one can show that $E$ satisfies a fourth-order polynomial and find $E$ as an explicit function of $\alpha, \beta, \gamma$ [11. By imposing the condition that the eigenvectors are normalisable (i.e. belong to $\left.L^{2}([0,1])\right)$ we obtain the discrete spectrum $E_{n}$ and can determine the coefficients $c_{1}$ and $c_{2}$. We impose conditions on $\alpha, \beta, \gamma, c_{1}$ and $c_{2}$ such that

$$
\int_{0}^{1} d z \frac{R(z)}{4} z^{\gamma-2}(1-z)^{-\gamma+\alpha+\beta-1} F^{2}(\alpha, \beta, \gamma, z)<\infty
$$


Looking at the asymptotic behavior of ${ }_{2} F_{1}(\alpha, \beta, \gamma, z)$ near $z=0$ and $z=1[1]{ }^{4}$, we obtain the following conditions

\begin{tabular}{|c|c|}
\hline$r_{0} \neq 0$ & $c_{1}=0, \gamma<1$ or $c_{2}=0, \gamma>1$ \\
\hline$r_{0}=0, r_{1} \neq 0$ & $c_{1}=0, \gamma<2$ or $c_{2}=0, \gamma>0$ \\
\hline$r_{0}=0, r_{1}=0$ & $c_{1}=0, \gamma<3$ or $c_{2}=0, \gamma>-1$ \\
\hline \multicolumn{2}{|c|}{ TABLE 2 condition $z=0$}
\end{tabular}

\begin{tabular}{|c|c|}
\hline$c_{1}=0$ & $\alpha-1 \in \mathbb{N}^{*}, \alpha+\beta-\gamma<0$ or $-1-\alpha+\gamma \in \mathbb{N}^{*}, \alpha+\beta-\gamma>0$ \\
\hline$c_{2}=0$ & $-\alpha \in \mathbb{N}^{*}, \alpha+\beta-\gamma>0$ or $\alpha-\gamma \in \mathbb{N}^{*}, \alpha+\beta-\gamma<0$ \\
\hline
\end{tabular}

TABLE 3. condition $z=1$

We have the discrete eigenvalues $\left(\alpha_{n}=-n ; n \in \mathbb{N}^{*}\right)$

(3.11) $\psi_{n}(s)=\left(z^{\prime}\right)^{-\frac{1}{2}} z^{\frac{\gamma_{n}}{2}}(z-1)^{\frac{-\gamma_{n}+\alpha_{n}+\beta_{n}+1}{2}} F\left(\alpha_{n}, \beta_{n}, \gamma_{n}, z\right)$

(3.12) $\gamma_{n}=1+\sqrt{1-r_{0} E_{n}-s_{0}}$

(3.13) $\alpha_{n}-\beta_{n}=-\sqrt{1-r_{2} E_{n}-s_{2}}$

(3.14) $\alpha_{n}+\beta_{n}=\gamma_{n}+\sqrt{1-\left(r_{0}+r_{1}+r_{2}\right) E_{n}-\left(s_{0}+s_{1}+s_{2}\right)}$

and the (normalised) eigenvectors

$$
\psi_{n}(s)=B_{n}\left(z^{\prime}\right)^{-\frac{1}{2}} z^{\frac{\gamma_{n}}{2}}(1-z)^{\frac{-\gamma_{n}-n+\beta_{n}+1}{2}} P_{n}^{\left(\gamma_{n}-1,-n+\beta_{n}-\gamma_{n}+1\right)}(1-2 z)
$$

with $B_{n}=\left[\left(\left(\frac{R(1)}{\alpha+\beta-\gamma}\right)+\left(\frac{r_{0}}{\gamma-1}\right)-\left(\frac{r_{2}}{\beta-\alpha}\right)\right) \frac{\Gamma(\gamma+n+1) \Gamma(\alpha+\beta-\gamma)}{n ! \Gamma(\beta-\alpha-n)}\right]^{-\frac{1}{2}}$ and $P_{n}^{(\gamma-1, \alpha+\beta-\gamma)}(2 z-1)$ the Jacobi polynomials.

3.4.2. Confluent hypergeometric potential. A similar construction can be achieved for the class of scaled confluent hypergeometric functions. The potential is given by

$$
Q(s)=\frac{S(z)-1}{R(z)}-\left(\frac{r_{1}}{z}-\frac{5}{4} \frac{\left(r_{1}^{2}-4 r_{2} r_{0}\right)}{R(z)}-r_{2}\right) \frac{z^{2}}{R(z)^{2}}
$$

with $R(z)=r_{2} z^{2}+r_{1} z+r_{0}>0$ and $S(z)=s_{2} z^{2}+s_{1} z+s_{0}$. The $z$ coordinate, lying in the interval $[0, \infty[$, is defined implicitly in terms of $s$ by the differential equation

$$
\frac{d z(s)}{d s}=\frac{2 z}{\sqrt{R(z)}}
$$

Example 3.4.2. The confluent Natanzon potential reduces to the Morse potential $Q(s)=\frac{-1+s_{0}+s_{1} e^{\frac{2 s}{\sqrt{r_{0}}}}+s_{2} e^{\frac{4 s}{\sqrt{r_{0}}}}}{r_{0}}$ for $r_{1}=r_{2}=0$, to the 3D oscillator $Q(s)=\frac{-\frac{3}{4}+s_{0}}{s^{2}}+\frac{s_{1}}{r_{1}}+\frac{s_{2} s^{2}}{r_{1}^{2}}$ for $r_{0}=r_{2}=0$ and to the Coulomb potential $Q(r)=\frac{-r_{2} s_{0}-2 s \sqrt{r_{2}} s_{1}-4 s^{2} s_{2}}{4 r_{2} s^{2}}$ for $r_{0}=r_{1}=0$.

$$
4_{2} F_{1}(\alpha, \beta, \gamma, z) \sim_{z \rightarrow 1} \Gamma(\gamma)\left((-1+z)^{-\alpha-\beta+\gamma}+\frac{\Gamma(-\alpha-\beta+\gamma)}{\Gamma(-\alpha+\gamma) \Gamma(-\beta+\gamma)}\right)
$$


By construction, the solution to the $\mathrm{CHE}$ potential is given in terms of the confluent hypergeometric function $F(\alpha, \beta, \gamma, z)$

$$
\psi(s, E)=z(s)^{\frac{\gamma}{2}} e^{-\frac{\omega z(s)}{2}}\left(z^{\prime}(s)\right)^{-\frac{1}{2}} F(\alpha, \beta, \gamma, \omega z(s))
$$

The parameters $\omega, \gamma, \alpha$ depend explicitly on the eigenvalue $E$ by

$$
\begin{aligned}
& \omega^{2}=-r_{2} E-s_{2} \\
& 2 \omega(\gamma-2 \alpha)=r_{1} E+s_{1} \\
& \gamma(2-\gamma)=r_{0} E+s_{0}
\end{aligned}
$$

Note that $\phi(z) \doteq F(\alpha, \beta, \gamma, \omega z(s))$ satisfies the differential equation [1]

$$
z \phi^{\prime \prime}(z)+(\gamma-\omega z) \phi^{\prime}(z)-\omega \alpha \phi(z)=0
$$

and the most general solution to (3.22) is generated by a two-dimensional vector space

$$
F(\alpha, \gamma, \omega z)=c_{1} M(\alpha, \gamma, \omega z)+c_{2}(\omega z)^{1-\gamma} M(1+\alpha-\gamma, 2-\gamma, \omega z)
$$

with $M(\alpha, \gamma, \omega z)$ the M-Whittaker function and $c_{1}$ and $c_{2}$ two arbitrary coefficients. By imposing that the eigenvectors are normalisable ((i.e. belong to $\left.L^{2}\left(\mathbb{R}^{+}\right)\right)$), we obtain the following conditions (see Table $2 \& 5$ ) which give the spectrum $E$ and the coefficients $c_{1}$ and $c_{2}{ }^{5}$

$$
\begin{array}{|c|c|}
\hline \alpha>2 & \text { no condition } \\
\hline \alpha \leq 2 & \begin{array}{c}
c_{1}=0,-1-\alpha+\gamma \in \mathbb{N}^{*} \\
\text { or } c_{2}=0,-\alpha \in \mathbb{N}^{*}
\end{array} \\
\hline
\end{array}
$$

TABLE 4. condition $z=\infty$

We have the discrete eigenvalues $\alpha_{n}=-n ; n \in \mathbb{N}$

$$
\begin{aligned}
\gamma_{n} & =1+\sqrt{1-r_{0} E_{n}-s_{0}} \\
\omega_{n} & =\sqrt{-r_{2} E_{n}-s_{2}} \\
2 n+1 & =\frac{r_{1} E_{n}+s_{1}}{2 \sqrt{-r_{2} E_{n}-s_{2}}}-\sqrt{1-r_{0} E_{n}-s_{0}}
\end{aligned}
$$

and the (normalized) eigenvectors

$$
\psi_{n}(s)=\frac{n !}{\left(\gamma_{n}\right)_{n}} z(s)^{\frac{\gamma_{n}}{2}} e^{-\frac{\omega_{n} z(s)}{2}}\left(z^{\prime}(s)\right)^{-\frac{1}{2}} L_{n}^{\gamma_{n}-1}\left(\omega_{n} z(s)\right)
$$

with $L_{n}^{\gamma_{n}-1}(z)$ the (generalized) Laguerre polynomial. In the following, we have listed classical solvable superpotentials and the corresponding solvable local volatility models and solvable instantaneous short-rate models (Table $5 \& 6$ ).

\footnotetext{
${ }^{5} M(\alpha, \beta, z) \sim_{z \rightarrow \infty} \frac{\Gamma(\beta)}{\Gamma(\alpha)} e^{z} z^{\alpha-\beta}\left(1+O\left(|z|^{-1}\right)\right)$
} 


\begin{tabular}{|c|c|c|}
\hline Superpotential & $W(s)$ & $\frac{\sigma(s)}{\sigma 0}$ \\
\hline Shifted oscillator & $a s+b$ & $e^{a s^{2}+2 b s}$ \\
\hline Coulomb & $a+\frac{b}{s}$ & $s^{b} e^{2 a s}$ \\
\hline Morse & $a+b e^{-\alpha s}$ & $e^{2\left(-\left(\frac{b}{\alpha e^{\alpha s}}\right)+a s\right)}$ \\
\hline Eckart & $a \operatorname{coth}(\alpha s)+b$ & $e^{2\left(b s+\frac{a \log (\sinh (\alpha s))}{\alpha}\right)}$ \\
\hline Rosen-Morse & $a t h(\alpha s)+b$ & $e^{2 b s+\frac{2 a}{\alpha} \ln (\cosh (\alpha))}$ \\
\hline 3D oscillator & $a s+\frac{b}{s}$ & $e^{a s^{2}+2 b \ln (s)}$ \\
\hline P-T I $\alpha>2 b$ & $a t a n(\alpha s)+b \operatorname{cotg}(\alpha s)$ & $e^{2\left(-\left(\frac{a \log (\cos (\alpha s))}{\alpha}\right)+\frac{b \log (\sin (\alpha s))}{\alpha}\right)}$ \\
\hline P-T II $\alpha>2 b$ & ath $(\alpha s)+b \operatorname{coth}(\alpha s)$ & $e^{2\left(\frac{a \log (\cosh (\alpha s))}{\alpha}+\frac{b \log (\sinh (\alpha s))}{\alpha}\right)}$ \\
\hline
\end{tabular}

\begin{tabular}{|c|c|c|}
\hline one-factor short-rate model & SDE & Superpotential \\
\hline Vasicek-Hull-White & $d r=k(\theta-r) d t+\sigma d W$ & Shifted Osc. $\left(a=\frac{\kappa}{2}, b=-\frac{\kappa \theta}{\sqrt{2} \sigma}\right)$ \\
\hline CIR & $d r=k(\theta-r) d t+\sigma \sqrt{r} d W$ & 3D Osc. $\left(a=\frac{\kappa}{4}, b=\frac{1}{2}-\frac{2 \theta \kappa}{\sigma^{2}}\right)$ \\
\hline Doleans & $d r=k r d t+\sigma r d W$ & (Constant $\left(W(s)=\frac{-2 \kappa+\sigma^{2}}{2 \sqrt{2} \sigma}\right)$ \\
\hline EV-BK & $d r=r(\eta-\alpha \ln (r)) d t+\sigma r d W$ & Shifted Osc. $\left(a=\frac{\alpha}{2}, b=\frac{\sqrt{2}\left(-2 \eta+\sigma^{2}\right)}{4 \sigma}\right)$ \\
\hline
\end{tabular}

TABLE 6. Example of solvable one-factor short-rate models

3.4.3. Natanzon hierachy and new solvable processes. We know that the Natanzon superpotential is related to the zero-eigenvector

$$
W_{\text {nat }}=-\partial_{s} \ln \left(\psi_{0}(s)\right)
$$

and the corresponding supercharge $A$ is

$$
\begin{aligned}
& A=\partial_{s}+W_{n a t}(s) \\
& =\frac{2 z(1-z)}{\sqrt{R}}\left(\partial_{z}-\frac{\gamma_{0}}{2 z}-\frac{\left(1+\alpha_{0}+\beta_{0}-\gamma_{0}\right)}{2(z-1)}-\frac{\alpha_{0} \beta_{0} F\left(1+\alpha_{0}, 1+\beta_{0}, 1+\gamma_{0}, z\right)}{\gamma_{0} F\left(\alpha_{0}, \beta_{0}, \gamma_{0}, z\right)}+\frac{z^{\prime-\frac{3}{2}} z^{\prime \prime}(s)}{2}\right)
\end{aligned}
$$

With a zero drift, the Natanzon superpotential corresponds to the diffusion process [3.3]

$$
\sigma^{(1)}(s)=\frac{\sigma_{0}^{(1)}}{\phi_{0}^{(1)}(s)^{2}}
$$

with $\sigma_{0}^{(1)}$ a constant of integration. Applying the results of section (3.2), we obtain that the driftless diffusion processes

$$
\sigma^{(m)}(s)=\frac{\sigma_{0}^{(m)}}{A_{m-1} \cdots A_{1} \phi_{m-1}^{(1)}(s)^{2}}
$$


are solvable (3.9). Using the fact that the (discrete) eigenvector $\phi_{m-1}^{(1)}(s)$ is a hypergeometric function and that the derivative of a hypergeometric function is a new hypergeometric function ${ }^{6}$ the action of $A_{m-1} \cdots A_{1}$ on $\phi_{m_{1}}^{(1)}(s)$ will result in a sum of $(m-1)$ hypergeometric functions, thus generalizing the solution found in [2].

\section{Gauge-Free Stochastic volatility models}

In this section, we try to identify the class of time-homogeneous stochastic volatility models which leads to an exact solution to the KBS equation. We assume that the forward $f$ and the volatility $a$ are driven by two correlated Brownian motions in the risk-neutral measure

$$
\begin{aligned}
& d f_{t}=a_{t} C\left(f_{t}\right) d W_{1} \\
& d a_{t}=b\left(a_{t}, f_{t}\right) d t+\sigma\left(a_{t}, f_{t}\right) d W_{2} \\
& d W_{1} d W_{2}=\rho d t
\end{aligned}
$$

with initial condition $a_{0}=a$ and $f_{0}=f$.

Using the definition for the connection (2.11), we obtain the Abelian connection ${ }^{7}$

$$
\begin{aligned}
& \mathcal{A}_{f}=-\frac{1}{2\left(1-\rho^{2}\right)} \partial_{f} \ln \left(\frac{C}{\sigma}\right)-\frac{\rho}{\left(1-\rho^{2}\right)}\left(\frac{b}{a C \sigma}-\frac{1}{2 C} \partial_{a} \frac{\sigma}{a}\right) \\
& \mathcal{A}_{a}=\frac{1}{\left(1-\rho^{2}\right)}\left(\frac{b}{\sigma^{2}}-\frac{1}{2} \partial_{a} \ln \left(\frac{\sigma}{a}\right)\right)
\end{aligned}
$$

Then, the field strength is

$$
\begin{aligned}
\mathcal{F}_{a f} & =\partial_{a} \mathcal{A}_{f}-\partial_{f} \mathcal{A}_{a} \\
& =\frac{1}{\left(1-\rho^{2}\right)}\left[\left(\partial_{a f} \ln (\sigma)-\partial_{f} \frac{b}{\sigma^{2}}\right)-\rho\left(\frac{1}{C} \partial_{a} \frac{b}{a \sigma}-\frac{1}{2 C} \partial_{a}^{2} \frac{\sigma}{a}+\frac{a}{2} \partial_{f}^{2} \frac{C}{\sigma}\right)\right]
\end{aligned}
$$

We will now assume that the connection is flat, $\mathcal{F}_{a f}=0$, meaning that the connection can be eliminated modulo a gauge transformation. In this case, the stochastic volatility model can thus be called a gauge-free model. This condition is satisfied for every correlation $\rho$ if and only if

$$
\begin{aligned}
& \partial_{a f} \ln (\sigma)=\partial_{f} \frac{b}{\sigma^{2}} \\
& \partial_{a} \frac{b}{a \sigma}-\frac{1}{2} \partial_{a}^{2} \frac{\sigma}{a}+\frac{a C}{2} \partial_{f}^{2} \frac{C}{\sigma}=0
\end{aligned}
$$

${ }_{7}^{6}{ }_{2} F_{1}^{\prime}(\alpha, \beta, \gamma, z)=\frac{\alpha \beta}{\gamma}{ }_{2} F_{1}(\alpha+1, \beta+1, \gamma+1, z)$ and $M^{\prime}(\alpha, \beta, z)=\frac{\alpha}{\beta} M(\alpha+1, \beta+1, z)$

$$
\begin{aligned}
\mathcal{A}^{f} & =-\frac{a^{2} C \sigma}{4} \partial_{f} \frac{C}{\sigma} \\
\mathcal{A}^{a} & =\frac{1}{2}\left(b-\frac{a \sigma}{2} \partial_{a} \frac{\sigma}{a}\right)
\end{aligned}
$$


Moreover, if we assume that $\sigma^{a}(a)$ is only a function of $a$ (this hypothesis is equivalent to assuming that the metric admits a Killing vector), the model is gauge-free if and only if

$$
\frac{b}{\sigma}=\frac{a}{2} \partial_{a} \frac{\sigma}{a}+a \phi(f)-\frac{a C(f)}{2} \partial_{f}^{2} C(f) \int \frac{a^{\prime} d a^{\prime}}{\sigma\left(a^{\prime}\right)}
$$

with $\phi(f)$ satisfying

$$
\partial_{f} \phi(f)=\frac{\partial_{f}\left(C \partial_{f}^{2} C\right)}{2} \int \frac{a^{\prime} d a^{\prime}}{\sigma\left(a^{\prime}\right)}
$$

This last equation is equivalent to $C(f) \partial_{f}^{2} C(f)=\beta$ with $\beta$ a constant and $\phi=\gamma$ a constant function. For $\beta=0$, the above equations simplify and we have

$$
\begin{aligned}
& C(f)=\mu f+\nu \\
& b(a)=a \sigma(a)\left(\gamma+\frac{1}{2} \partial_{a} \frac{\sigma(a)}{a}\right)
\end{aligned}
$$

with $\mu, \nu, \gamma$ constant. The gauge-free condition has therefore imposed the functional form of the drift term. When the volatility function is fixed respectively to a constant (Heston model), a linear function (geometric Brownian model) and a quadratic function (3/2-model) in the volatility, one obtains the correct (mean-reversion) drift $^{8}$ (see Table 7 )

\begin{tabular}{|c|c|c|}
\hline name & $\sigma(a)$ & $S D E$ \\
\hline Heston & $\sigma(a)=\eta$ & $d v=\sqrt{\delta}(2 v \gamma+\eta(\eta-1)) d t+2 \eta \sqrt{\delta} \sqrt{v} d W_{2}$ \\
\hline Geometric Brownian & $\sigma(a)=\eta a$ & $d v=\sqrt{\delta}\left(2 \eta \gamma v^{\frac{3}{2}}+\eta^{2} v\right) d t+2 \sqrt{\delta} \eta v d W_{2}$ \\
\hline $3 / 2$-model & $\sigma(a)=\eta a^{2}$ & $d v=2 \sqrt{\delta} \eta(\eta+\gamma) v^{2} d t+2 \sqrt{\delta} \eta v^{\frac{3}{2}} d W_{2}$ \\
\hline
\end{tabular}

TABLE 7. Examples of Gauge free stochastic volatility models with $d f=\delta(\mu+\nu f) \sqrt{v} d W_{1}^{\prime}$.

The gauge transformation eliminating the connection is then

$$
\Lambda(f, a)=-\frac{\rho^{f f}}{2} \ln (C(f))+\alpha \rho^{f a} \int^{f} \frac{d f^{\prime}}{C\left(f^{\prime}\right)}+\left(\rho^{f f} \alpha-\frac{\rho^{a f}}{2} \partial_{f} C\right) \int^{a} \frac{a^{\prime} d a^{\prime}}{\sigma\left(a^{\prime}\right)}
$$

Finally, plugging the expression for $C(f)$ and $b(a)$ into (2.12), we find that the function $Q$ is ${ }^{9}$

$$
Q=A a^{2}+B \sigma^{2} \partial_{a} \frac{a}{\sigma}
$$

The Black-Scholes equation for a Vanilla option $\mathcal{C}(a, f, \tau=T-t)$ (with strike $K$ and maturity $T$ ) satisfied by the gauge transformed function $\mathcal{C}^{\prime}(a, f, \tau)=e^{\Lambda(f, a)} \mathcal{C}(a, f, \tau)$ is

\footnotetext{
${ }^{8}$ In order to obtain the correct number of parameters, one needs to apply a change of local time $d t=\delta d t^{\prime}$, $d W_{1,2}=\sqrt{\delta} d W_{1,2}^{\prime}$

${ }^{9} A$ and $B$ are two constants given by

$$
\begin{aligned}
& A=\frac{1}{2}\left(-\frac{1}{2} \rho^{f f} \partial_{f} C+\alpha \rho^{a f}\right)^{2}+\frac{1}{2} \rho^{f f} C^{2} \partial_{f}^{2} \ln (C)+\rho\left(-\frac{1}{2} \rho^{f f} \partial_{f} C+\alpha \rho^{a f}\right)\left(\alpha \rho^{f f}-\frac{\rho^{a f}}{2} \partial_{f} C\right)+\frac{1}{2}\left(\rho^{f f} \alpha-\frac{\rho^{a f}}{2} \partial_{f} C\right)^{2} \\
& B=-\frac{1}{2}\left(\rho^{f f} \alpha-\rho^{a f} \frac{\partial_{f} C}{2}\right)
\end{aligned}
$$
}




$$
\partial_{\tau} \mathcal{C}^{\prime}(a, f, \tau)=\Delta \mathcal{C}^{\prime}(a, f, \tau)+Q(a) \mathcal{C}^{\prime}(a, f, \tau)
$$

with the initial condition $\mathcal{C}^{\prime}(a, f, \tau=0)=e^{\Lambda(f, a)}(f-K)^{+}$. In the coordinates $q(f)=\iint^{f} \frac{d f^{\prime}}{C\left(f^{\prime}\right)}$ and $a$, the Laplace-Beltrami operator is given by

$$
\Delta=\frac{a \sigma}{2}\left(\frac{a}{\sigma} \partial_{q}^{2}+2 \rho \partial_{a q}+\partial_{a} \frac{\sigma}{a} \partial_{a}\right)
$$

Applying a Fourier transformation according to $q, \mathcal{C}^{\prime}(q, a, \tau)=\mathcal{F C}^{\prime}(f, a, \tau)$, we obtain

$$
\partial_{\tau} \mathcal{C}^{\prime}(a, q, \tau)=\frac{a \sigma}{2}\left(-k^{2} \frac{a}{\sigma}+2 i k \rho \partial_{a}+\partial_{a} \frac{\sigma}{a} \partial_{a}\right) \mathcal{C}^{\prime}(a, q, \tau)+Q(a) \mathcal{C}^{\prime}(a, q, \tau)
$$

with the initial condition $\mathcal{C}^{\prime}(a, q, \tau=0)=\mathcal{F}\left[e^{\Lambda(f, a)}(f-K)^{+}\right]$. Using a spectral decomposition $\mathcal{C}^{\prime}(a, q, \tau)=\sum_{n} \phi_{n k}(a)\left(\mathcal{C}^{\prime}(a, q, \tau=0), \phi_{n k}\right) e^{-E_{n k} \tau}$ (with (.,.) the scalar product on $\left.L^{2}\right)$, the eigenvectors $\phi_{n k}(a)$ satisfy the equation

$$
-E_{n k} \phi_{n k}(a)=\frac{a \sigma}{2}\left(-k^{2} \frac{a}{\sigma}+2 i k \rho \partial_{a}+\partial_{a} \frac{\sigma}{a} \partial_{a}\right) \phi_{n k}(a)+Q(a) \phi_{n k}(a)
$$

This equation (4.21) can be further simplified by applying a Liouville transformation consisting in a gauge transformation and a change of variable [21]

$$
\begin{aligned}
\psi_{n k}(s) & =\left(\frac{\sigma}{a}\right)^{\frac{1}{2}} e^{i k \rho \int^{a} \frac{a^{\prime}}{\sigma\left(a^{\prime}\right)} d a^{\prime}} \phi_{n k}(a) \\
\frac{d s}{d a} & =\frac{\sqrt{2}}{\sigma(a)}
\end{aligned}
$$

$\psi_{n k}(s)$ satisfies a Schrodinger equation

$$
\psi_{n k}^{\prime \prime}(s)+\left(E_{n k}-J(s)\right) \psi_{n k}(s)=0
$$

with the scalar potential

$$
J(s)=Q(a)-\frac{k^{2} a^{2}}{2}+\frac{4 a^{4} k^{2} \rho^{2}-3 \sigma(a)^{2}+a^{2} \sigma^{\prime}(a)^{2}+2 a \sigma(a)\left(\sigma^{\prime}(a)-a \sigma^{\prime \prime}(a)\right)}{8 a^{2}}+\frac{1}{2}\{a, s\}
$$

and where the curly bracket denotes the Schwarzian derivative of $a$ with respect to $s$

$$
\{a, s\}=\left(\frac{a^{\prime \prime}(s)}{a^{\prime}(s)}\right)^{\prime}-\frac{1}{2}\left(\frac{a^{\prime \prime}(s)}{a^{\prime}(s)}\right)^{2}
$$

The $2 d$ partial differential equation corresponding to our original KBS equation for our stochastic volatility model has therefore been reduced via a change of coordinates and gauge transformations to a Schrodinger equation with a scalar potential $J(s)$. The stochastic volatility model is therefore 
solvable in terms of hypergeometric functions if the potential $J(s)$ belongs to the Natanzon class. The solution is given (in terms of the eigenvectors $\psi_{n k}$ ) by

$$
\mathcal{C}(a, f, \tau)=e^{-\Lambda(a, f)} \mathcal{F}^{-1}\left[\sum_{n} \psi_{n k}(s(a))\left(\mathcal{F}\left[e^{\Lambda(f, a)}(f-K)^{+}\right], \psi_{n k}(s(a))\right) e^{-E_{n k} \tau}\right]
$$

Let us examine classical examples of solvable stochastic volatility model and show that the corresponding potentials $J(s)$ correspond to the Natanzon class (see Table 8).

\begin{tabular}{|c|c|c|}
\hline name & potential & $J(s)$ \\
\hline Heston & 3D osc. & $J(s)=\frac{-3+4 B s^{2} \eta+s^{4} \eta^{2}\left(2 A+k^{2}\left(-1+\rho^{2}\right)\right)}{4 s^{2}}$ \\
\hline Geometric Brownian & Morse & $J(s)=\frac{-\eta^{2}+4 e^{\sqrt{2} s \eta}\left(2 A+k^{2}\left(-1+\rho^{2}\right)\right)}{8}$ \\
\hline $3 / 2$-model & Coulombian & $J(s)=\frac{8 A+\eta(-8 B+\eta)+4 k^{2}\left(-1+\rho^{2}\right)}{4 s^{2} \eta^{2}}$ \\
\hline
\end{tabular}

TABLE 8. Stochastic volatility models and potential $J(s)$

We present here a new example of solvable stochastic volatility model which corresponds to the Posh-Teller I potential.

Example 4.0.3 (Posh-Teller I). For a volatility function given by $\sigma(a)=\alpha+\eta a^{2}$, the potential is given by a Posh-Teller I potential ${ }^{10}$

$J(s)=\frac{\alpha\left(4\left(-2 A+k^{2}+4 B \eta-k^{2} \rho^{2}\right)-3 \eta^{2} \csc \left(\frac{s \sqrt{\alpha} \sqrt{\eta}}{\sqrt{2}}\right)^{2}+\left(8 A+\eta(-8 B+\eta)+4 k^{2}\left(-1+\rho^{2}\right)\right) \sec \left(\frac{s \sqrt{\alpha} \sqrt{\eta}}{\sqrt{2}}\right)^{2}\right)}{8 \eta}$

\section{Conclusion}

We have shown how to use supersymmetric methods to generate new solutions to the Kolmogorov \& Black-Scholes equation (KBS) for one-dimensional diffusion processes. In particular, by applying a supersymmetric transformation on the Natanzon potential, we have generated a hierarchy of new solvable processes. Then, we have classified the stochastic volatility models which admit a flat Abelian connection (with one Killing vector). The two-dimensional KBS equation has been converted into a Schrodinger equation with a scalar potential. The models for which the scalar potential belongs to the Natanzon class are solvable in terms of hypergeometric functions. This is the case for the Heston model, the geometric brownian model and the 3/2-model. A new solution with a volatility of the volatility $\sigma(a)=\alpha+\eta a^{2}$, corresponding to the Posh-Teller I, has been presented.

\section{ACKNOWLEDGEMENTS}

I would like to thank Dr. S. Ribault for useful comments and discussions and Dr. C. Waite for a careful reading.

\footnotetext{
${ }^{10} \csc (z) \equiv \frac{1}{\sin (z)}$ and $\sec (z) \equiv \frac{1}{\cos (z)}$
} 


\section{REFERENCES}

[1] Handbook of Mathematical Functions With Formulas, Graphs, and Mathematical Tables (AMS55), http://www.convertit.com/Go/ConvertIt/Reference/AMS55.ASP?Res=150

[2] Albanese, C., Campolieti, G., Carr, P., Lipton, A., Black-Scholes Goes Hypergeometric, Risk magazine

[3] Albanese, C. , Kuznetsov, A., Transformations of Markov Processes and Classification Scheme for Solvable Driftless Diffusions

[4] Albanese, C. , Kuznetsov, A., Unifying the Three Volatility Models

[5] Albanese, C. , Kuznetsov, A., Hauviller, P., A Clasification Scheme for Integrable Diffusions

[6] Bonneau, G., Faraut, J., Valent, G., Self-adjoint Extensions of Operators and the Teaching of Quantum Mechanics, Am.J.Phys. 69 (2001) 322, quant-ph/0103153

[7] Carr, P., Lipton, A., Madan, D., Reduction Method for Valuing Derivative Securities, Working paper, http://www.math.nyu.edu/research/carrp/papers/pdf/symmetry17.pdf

[8] F. Cooper, A. Khare, U. Sukhatme, Supersymmetry and Quantum Mechancis, hep-th/9405029

[9] Cox, J.C, Notes on Option Pricing I: Constant Elasticity of Variance Diffusions, Journal of Portfolio Management, 22, 15-17, 1996.

[10] Cox, J.C, Ingersoll, J.E, Ross, S.A, A Theory of the Term Structure of Interest Rates, Econometrica, 53, 385-407, 1985.

[11] Grosche, C. , The General Besselian and Legendrian Path Integrals, J. Phys. A: Math. Gen. 29 No 8 (1996)

[12] Heston, S.: A closed-for solution for options with stochastic volatility with applications to bond and currency options, review of financial studies, 6, 327-343.

[13] Hull, J., White, A., Pricing Interest Rate Derivative Securities, The Review of Financial Studies, 3, 573-592.

[14] Ito, K, McKean, H., Diffusion Processes and their Sample paths, Springer, Berlin.

[15] Junker, G., Quantum and Classical Dynamics: Exactly Solvable Models by Supersymmetric Methods, quant-ph/9810070

[16] Kuznetsov, A., Solvable Markov Processes, Phd. Thesis (2004).

[17] Krylov, G., On Solvable Potentials for One Dimensional Schrodinger and Fokker-Planck Equations

[18] Lewis, A., Option valuation under Stochastic volatility, Finance Press (2000), CA.

[19] Lipton, A., Mathematical Methods for Foreign Exchange, World Scientific (2001).

[20] Linetsky, V., The Spectral Decomposition of the Option Value, To appear International Journal of Theoretical and Applied Finance.

[21] Milson, R., On the Liouville Tranformation and Exactly-Solvable Schrodinger Equations, solv-int/9706007

[22] Natanzon, G.A, Study of one-dimensional Schrödinger equation generated from the hypergeometric equation, Vestnik Leningradskogo Universiteta, 10:22, 1971, ArXiv:physics/9907032

[23] Reed, M, Simon, B., Methods of Mathematical Physics, vol. 2, Academic Press.

E-mail address: phl@hotmail.com 